\title{
Mecanismos de construcción-destrucción de patrones macroestructurales en microclimas como sistemas complejos
}

\author{
L. SÁNCHEZ-MUÑOZ, O. ROVER*, J. SANZ**, J. GARCÍA-GUINEA*** \\ CIEMAT, Avda. Complutense 22, 28040 Madrid \\ *CRSCM-CNRS (Orléans, Francia) \\ ** Instituto Ciencia de Materiales de Madrid (CSIC) \\ ***Museo Nacional de Ciencias Naturales (CSIC)
}

\begin{abstract}
Se han estudiado estructuras de feldespatos potásicos triclínicos (microclinas) intercrecidos con feldespatos sódicos en texturas pertíticas procedentes de pegmatitas graníticas a diferentes escalas de observación, mediante microscopía de luz transmitida (MLT), difracción de rayos-X (DRX), resonancia magnética nuclear de alta resolución del núcleo ${ }^{27} \mathrm{Al}$ (RMN), y mapas químicos de contenido en Na por microsonda electrónica (ME). Las pertitas pegmatíticas son sistemas complejos en cuyos feldespatos potásicos se han identificado dos periodos genéticos independientes de recristalización durante el enfriamiento geológico. En el primer periodo, en la estructura mineral se produce la construcción gradual de patrones macroscópicos regulares pseudoperiódicos, como consecuencia del efecto combinado de un ordenamiento local no-ergódico en la distribución $\mathrm{Si} / \mathrm{Al}$ y de un efecto cooperativo global de la estructura del cristal. Desde las interfases incoherentes con el feldespato sódico surgen frentes de transformación a modo de avalanchas que pueden coalescer longitudinalmente y yuxtaponerse transversalmente para auto-ensamblarse, y finalmente evolucionar hasta formar dominios-macla (leyes Albita y Periclina), como unidad cristalina de construcción de la macroestructura. Estos procesos de auto-organización se activan cuando el sistema está abierto a la circulación de moléculas de agua catalizadoras para el ordenamiento de la red, al tiempo que existe una estimulación externa por tensiones tectónicas de cizalla. Durante el segundo periodo se destruyen los patrones estructurales creados previamente, por: (i) maclados de relajación y deformación, (ii) engrosamientos de dominios por disolución-reprecipitación debidos a interacciones con fluidos acuosos a baja temperatura, (iii) reorientaciones de dominiosmacla según ley Periclina hacia orientaciones de ley Albita.
\end{abstract}

Palabras clave: microclina, orden Si/Al, dominios-macla, macroestructuras, auto-organización, sistemas complejos.

Mechanism of construction-destruction of macrostructural patterns in perthitic microcline as complex systems.

The structures of microcline perthites collected from granitic pegmatites have been studied at different observation scales using optical microscopy (OM), X-ray diffraction (XRD), ${ }^{27}$ Al MASS-NMR spectroscopy, and electron microprobe (EMPA) with Na mappings. Pegmatite perthites are identified as complex systems. During the cooling process, K-rich feldspars display two separated genetic periods of recrystallization, each one with variable intensity along different samples. During the first period, in the mineral structure a progressive construction of macrostructural pseudo-periodic patterns is produced. These regular patterns emerge due to a combined mechanism of non-ergodic Si / Al local ordering and a global cooperative effect of the crystal structure. From the incoherent $\mathrm{Na} / \mathrm{K}$ interfaces arise transformation fronts (avalanches) which can coalesce longitudinally and juxtapose transversally to self-assemble into irregular domains. Finally, they can evolve up to create regular twin-domains (Albite and Pericline laws) as the crystal unit of the macrostructural building. This selforganization process is activated when the system is open to water circulation acting as a catalyst of $\mathrm{Al} / \mathrm{Si}$ ordering in the feldspar lattice linked with an external stimulation by shear tectonic stress. During the second period the structural patterns, previously created, are destroyed by (i) relaxation and deformation twinning mechanisms, (ii) coarsening of twin-domains produced by dissolution-precipitation due to aqueous fluids interactions at low temperature, (iii) reorientation of Periclinelaw twin-domains to the Albite-law orientation.

Keywords: microcline, Si/Al order, twin-domains, macrostructures, self-organization, complex system

\section{INTRODUCCIÓN}

Las pertitas de las pegmatitas graníticas son materias primas cerámicas compuestas por dos fases minerales: el feldespato potásico $(\mathrm{K}>>\mathrm{Na}) \mathrm{AlSi}_{3} \mathrm{O}_{8}$ es mayoritario y actúa como matriz del feldespato sódico $\mathrm{NaAlSi}_{3} \mathrm{O}_{8}$ que lo hace como huésped (1). El origen de las pertitas se debe a que el feldespato potásico cristalizado a alta temperaturas forma disolución sólida con el feldespato sódico, pero sufren procesos de exsolución e interacción con fluidos acuosos ricos en sodio durante el enfriamiento. Las interfases, o superficies limítrofes de separación entre las dos fases, son semicoherentes en las texturas micropertíticas que están compuestas por lamelas finas de feldespatos sódicos y se han formado por exsolución a alta temperatura. Sin embargo, cuando desciende la temperatura se produce la interacción con fluidos acuosos dando lugar a disolución de algunas lamelas y engrosamiento de otras para dar lugar a las texturas macropertíticas con venas de feldespato sódico, caracterizadas por interfases incoherentes (2-3).

Los cristales de feldespato sódico-potásico formados a altas temperaturas a partir de la cristalización de fundidos ígneos 
tienen estructura con simetría de celda unidad monoclínica $\mathrm{C} 2 / \mathrm{m}$ y desorden atómico en la distribución Si/Al. Estos cationes se distribuyen en dos posiciones tetraédricas no equivalentes llamadas $\mathrm{T}_{1}$ y $\mathrm{T}_{2}$. En ambiente volcánico el enfriamiento rápido inhibe grandes cambios estructurales en el cristal (a excepción de procesos de exsolución $\mathrm{Na} / \mathrm{K}$ ) apareciendo a temperatura ambiente cristales con simetría monoclínica, esto es, se observa la fase de alta temperatura llamada sanidina. Sin embargo, debido al enfriamiento lento en el ambiente plutónico se produce un cambio de fase a una temperatura de transformación $\mathrm{T}_{\mathrm{s} / \mathrm{m}}$ de unos $475{ }^{\circ} \mathrm{C}$ en microclina o fase de baja temperatura, que tiene simetría de celda unidad triclínica $\mathrm{C} 1$ y cuatro posiciones tetraédricas $\mathrm{T}_{10^{\prime}}$ $\mathrm{T}_{1 \mathrm{~m}^{\prime}} \mathrm{T}_{2 \mathrm{o}} \mathrm{y} \mathrm{T}_{2 \mathrm{~m}}(2)$

Durante el enfriamiento, cuando no actúan los factores cinéticos de catalización de reacciones de ordenamiento $\mathrm{Si} / \mathrm{Al}$ (fluidos acuosos y presiones tectónicas dirigidas), la estructura de la microclina no tiene capacidad cinética para re-equilibrarse, incluso para velocidades de unos pocos grados centígrados por millón de años, y se mantiene con la distribución $\mathrm{Si} / \mathrm{Al}$ desordenada y con baja triclinicidad en la celda unidad hasta la temperatura ambiente. Esto es debido a que el sistema evoluciona poco según un régimen estático (4) porque la capacidad de recristalización es muy limitada, y por tanto se conserva en gran medida el material cristalino formado inmediatamente después de la transformación de la sanidina en microclina. Estas microclinas primitivas constan de estructuras moduladas compuestas por nanodominios irregulares con límites mal definidos (microestructura "tweed"), empleando el término microclina alta (o ortoclasa) para dicha variedad (refs. 5 y 6). Sin embargo, cuando actúan los factores cinéticos se activa un régimen dinámico de recristalización (4), que transforma la microclina alta en variedades más ordenadas por aumento del orden $\mathrm{Si} / \mathrm{Al}$ a nivel local que se traduce en incremento de la triclinicidad de la celda unidad. En el caso extremo, aparecen estructuras que a nivel local presentan todo el $\mathrm{Al}$ ordenado en la posición $\mathrm{T}_{10}$ y máxima triclinicidad, caracterizadas por macroestructuras en dominios-macla observables a la escala óptica, usando el término microclina baja para dicha variedad $(5,7)$. Las microclinas intermedias son la variedad de microclina con características comprendidas entre los extremos alta y baja (8).

Estas estructuras de dominios-macla a gran escala se han interpretado tradicionalmente desde los primeros trabajos de Laves (9) como maclados de transformación, esto es, como consecuencia de la pérdida local de los elementos de simetría en la celda unidad durante la transformación monoclínico-triclínico $\mathrm{C} 2 / \mathrm{m} \rightarrow \mathrm{C} \overline{1}$. Las microclinas pueden presentar dominios que se relacionan con cuatro variantes orientacionales, dos a dos por un plano espejo " $\mathrm{m}$ " y un eje binario "2". La ley de la Albita relaciona dominios cristalinos mediante una reflexión según un plano paralelo a (010), que constituye tanto el plano de macla como el plano de composición. En la ley de la Periclina la orientación relativa de los dominios responde a un eje binario paralelo a [010], que constituye el eje de macla, mientras que el plano de composición es un plano irracional llamado sección rómbica paralelo a [010] $(2,7,9)$.

Sin embargo, existen evidencias relativas a las características de las estructuras de maclado de las microclinas que apuntan hacia un origen más complejo que la simple hipótesis del maclado de transformación $(7,10)$. En este trabajo se analiza el origen y evolución de las macroestructuras en dominios-macla formadas como consecuencia de la transformación microclina alta-baja, utilizando datos obtenidos a distintas escalas de observación. Para ello se han usado pertitas de pegmatitas graníticas formadas en muy diversos ambientes geológicos caracterizadas por haberse generado en distintos regímenes de evolución durante el enfriamiento.

\section{METODOLOGÍA EXPERIMENTAL}

En este trabajo se presenta un análisis de las macroestructuras representativas de un gran número de materiales estudiados en detalle en una publicación precedente en el BSECV, donde se incluyen las características geológicas de las pegmatitas muestreadas, los datos de difracción de rayos- $X$ y la composición química por fluorescencia de rayos$X$ (4). Las fotomicrografías de microclinas han sido obtenidas por microscopía óptica de transmisión de luz polarizada en secciones aproximadamente paralelas al plano (001) y con nícoles cruzados, debido a que permiten: i) identificar la variedad de microclina en función del ángulo de extinción óptica, ya que el grado de triclinicidad está linealmente relacionado con el ángulo de extinción sobre (001) de la traza de (010) (11); ii) observar distintos contrastes de dominios con diferente orientación maclados según las leyes de la Albita y de la Periclina, que son aproximadamente perpendiculares entre ellos y a esta sección de observación; iii) identificar las variedades macroestructurales en base a contrastes por distinto ángulo de extinción y por la distribución de las paredes de dominio; iv) observar las texturas pertíticas que se dan en todas las muestras. Las fotomicrografías se han obtenido sin uso de la lente condensadora y en condiciones de iluminación óptimas para obtener el máximo contraste de extinción para regiones estructuralmente diferentes.

Los mapas químicos mostrados en las Fig. $5 \mathrm{~b}$ y $7 \mathrm{~b}$ se han obtenido según esta misma orientación por microsonda electrónica utilizando un equipo SX-50, con el programa ZAF para corregir los efectos de matriz, construyendo las imágenes a partir de 25000 análisis puntuales en cada caso empleando la $\mathrm{K}_{\alpha}$ del $\mathrm{Na}$. Se utilizaron varias condiciones experimentales para minimizar la pérdida de $\mathrm{Na}$ y maximizar la obtención de señal y finalmente se trabajo a $15 \mathrm{kV}$ y $100 \mathrm{nA}$ en la imagen de alta resolución (Fig. 6b) y a $15 \mathrm{kV}$ y $50 \mathrm{nA}$ en la imagen de baja resolución (Fig. 8b). El tamaño de la sonda fue aproximadamente de $1 \mu \mathrm{m}^{3}$.

Los datos estructurales relativos al grado de ordenamiento $\mathrm{Si} / \mathrm{Al}$ se han obtenido mediante espectroscopía de resonancia magnética nuclear (RMN) de alta resolución del núcleo ${ }^{27} \mathrm{Al}$, en condiciones de alta resolución utilizando la técnica de rotación al ángulo mágico a $54^{\circ} 44^{\prime}$ respecto del campo magnético externo. Los espectros de muestras en polvo fueron tomados a $104.24 \mathrm{MHz}$ en un espectrómetro equipado con unidad de transformada de Fourier. La frecuencia de giro estuvo en el rango de 4000 a 5000 cps. La duración de los impulsos $(\pi / 2)$ utilizados en la irradiación de la muestra fue $2 \mu \mathrm{s}$. El intervalo de tiempo entres sucesivas acumulaciones utilizado fue de 5 $\mathrm{s}$ con objeto de minimizar los efectos de saturación en cada señal. Como patrón externo fue usada una disolución de $\mathrm{Cl}_{3} \mathrm{Al}$. El número de acumulaciones estuvo comprendido entre 200 y 400. El error medio estimado en la determinación del valor del desplazamiento químico en la señal fue de 0.5 ppm. 


\section{RESULTADOS}

En la Fig. 1 se presentan los espectros de ${ }^{27} \mathrm{Al} \mathrm{RMN}$ de una microclina ordenada cercana a máximo orden local $\mathrm{Si} /$ Al (muestra GcI), una microclina desordenada próxima al

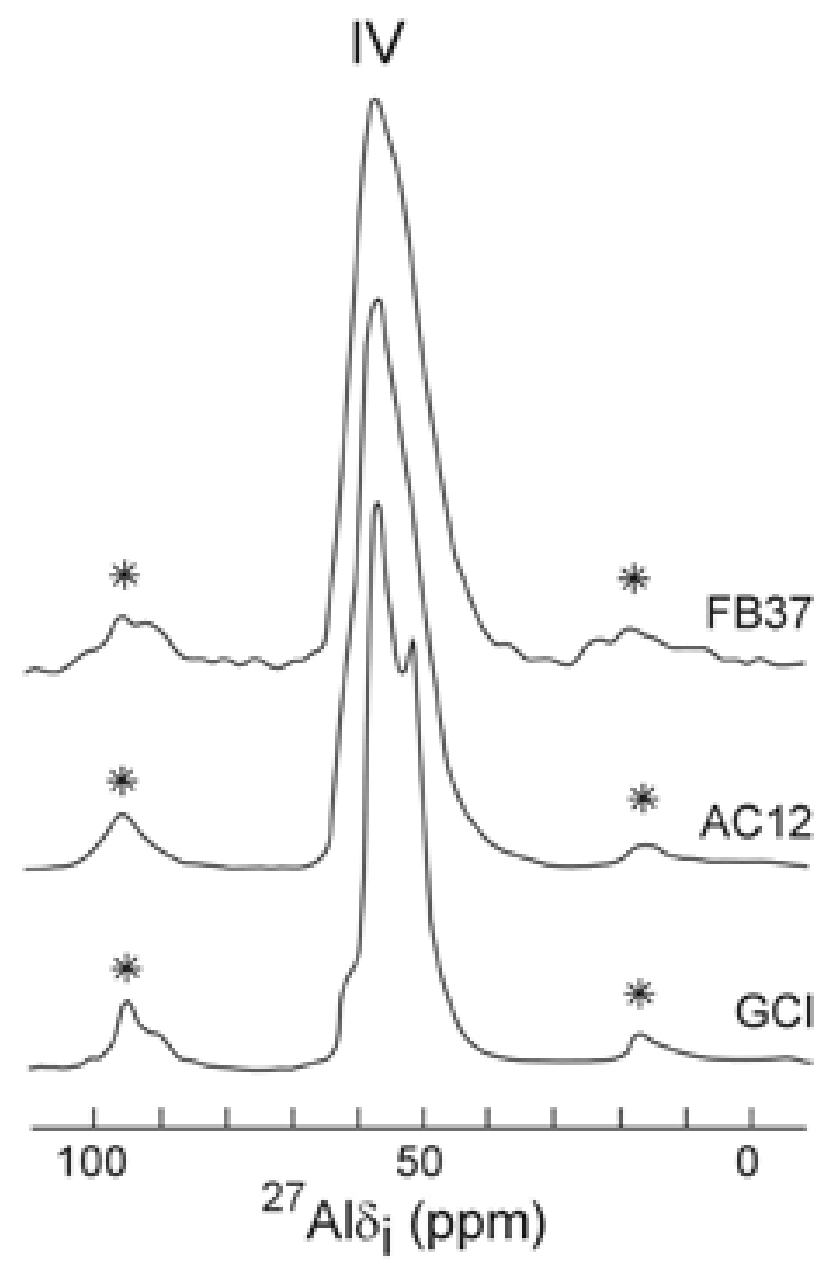

Fig. 1.- Espectros de ${ }^{27} \mathrm{Al}$ de resonancia magnética nuclear por el método de rotación con el ángulo mágico mostrando la transición central $1 / 2 \rightarrow-1 / 2$ como señal más intensa y las bandas laterales de rotación con asteriscos. Se presentan los espectros de tres muestras de microclina ordenada $(\mathrm{GcI})$, microclina transicional (Ac12) y microclina desordenada (FB37)

máximo desorden (muestra FB37) y una microclina transicional (muestra Ac12). En la Tabla I se encuentran los valores en ppm de la posición $\mathrm{p}_{\mathrm{Al}}$ del máximo de la transición central $1 / 2 \rightarrow-$ $1 / 2$, y su anchura $a_{A 1}$ en ppm obtenidos a partir de los espectros de ${ }^{27} \mathrm{Al}$. Los espectros están formados por tres señales a unas 56,51 y 46 ppm relacionadas con $\mathrm{Al}$ en la posición $\mathrm{T}_{1 \mathrm{o}}$ y una cuarta señal a unas $61 \mathrm{ppm}$ debida a $\mathrm{Al}$ en las tres restantes posiciones $\mathrm{T}_{1 \mathrm{~m}}, \mathrm{~T}_{20} \mathrm{y} \mathrm{T}_{2 \mathrm{~m}}$. A medida que aumenta el desorden la cuarta señal aumenta de intensidad y desplaza el máximo a valores más altos en el desplazamiento químico, al tiempo que los poliedros tetraédricos se deforman y cambian las constantes cuadrupolares. El resultado combinado de los dos efectos es el aumento de la anchura total de la señal, siendo este valor por tanto representativo del orden $\mathrm{Si} / \mathrm{Al}$ local medio en la muestra. En la Fig. 2 se representa un diagrama de correlación
TABLA I. VALORES DE POSICIÓN A Y ANCHURA A DE LA TRANSICIÓN CENTRAL DEL ESPECTRO 27AL RMN Y DEL PARÁMETRO S CALCULADO POR DRX

\begin{tabular}{|c|c|c|c|}
\hline Muestra & $\mathrm{p}_{\mathrm{Al}}(\mathrm{ppm})$ & $\mathrm{a}_{\mathrm{Al}}(\mathrm{ppm})$ & $\mathrm{S}_{2}(\mathrm{DRX})$ \\
\hline FB9 & 56.13 & 10.65 & 0.035 \\
\hline FB10 & 55.72 & 10.63 & 0.021 \\
\hline FB12 & 55.85 & 10.34 & 0.024 \\
\hline FB18 & 55.73 & 10.23 & 0.027 \\
\hline FB19 & 56.16 & 9.77 & 0.048 \\
\hline FB29 & 56.45 & 11.11 & 0.021 \\
\hline FB31 & 56.54 & 11.47 & 0.018 \\
\hline FB32 & 56.51 & 11.12 & 0.034 \\
\hline FB33 & 56.40 & 11.22 & 0.033 \\
\hline FB14 & 56.33 & 10.39 & 0.032 \\
\hline FB22 & 56.56 & 11.73 & 0.017 \\
\hline FB28 & 56.16 & 10.01 & 0.035 \\
\hline FB37 & 56.71 & 11.21 & 0.013 \\
\hline En1 & 55.99 & 8.91 & 0.075 \\
\hline En2 & 55.92 & 10.42 & 0.028 \\
\hline En7 & 55.95 & 9.75 & 0.030 \\
\hline En8 & 55.89 & 9.29 & 0.066 \\
\hline En11 & 56.10 & 10.09 & 0.028 \\
\hline En13 & 55.74 & 10.42 & 0.020 \\
\hline En17 & 56.33 & 10.34 & 0.024 \\
\hline En18 & 56.13 & 10.06 & 0.051 \\
\hline Ac3 & 56.15 & 9.02 & 0.076 \\
\hline $\mathrm{F} 2 \mathrm{~b}$ & 55.98 & 9.70 & 0.049 \\
\hline Ac12 & 56.07 & 8.78 & 0.090 \\
\hline Ac6 & 55.71 & 9.52 & 0.060 \\
\hline Dlr1 & 56.00 & 8.26 & 0.137 \\
\hline GcI & 55.95 & 8.49 & 0.137 \\
\hline GcF2 & 55.90 & 8.33 & 0.148 \\
\hline
\end{tabular}

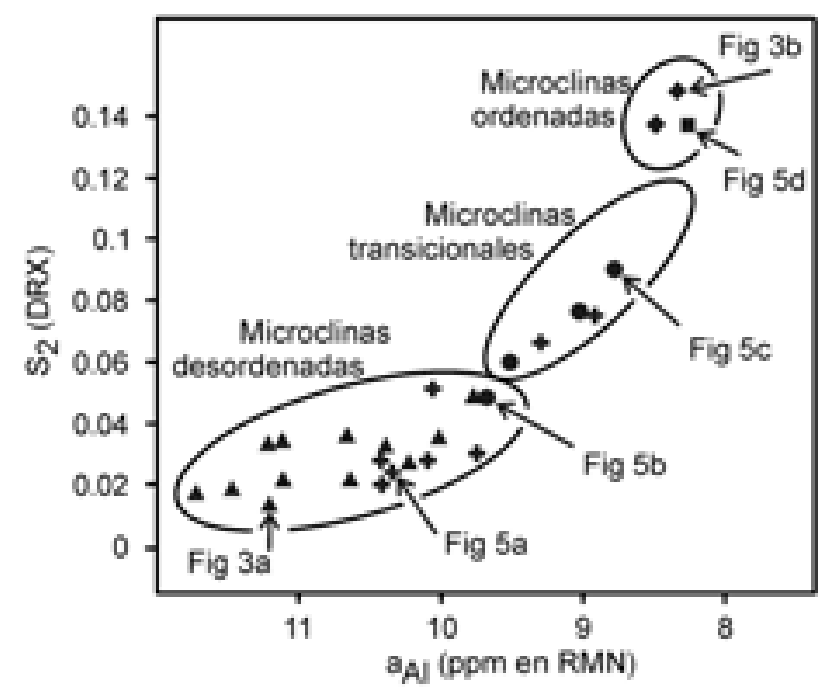

Fig. 2.-Diagrama de correlación estructural de microclinas con representación del grado de ordenamiento $\mathrm{Si} / \mathrm{Al}$ mediante el parámetro $S_{2}$ de DRX y a $a_{\mathrm{Al}} \mathrm{o}$ anchura de los espectros ${ }^{27} \mathrm{Al}$ de $\mathrm{RMN}$. Se han representado tres regiones correspondientes a microclinas ordenadas, transicionales y desordenadas, así como la posición en el diagrama de las muestras utilizadas en las figuras siguientes, esto es, FB37, En17, F2b, Ac12 y Dlr1. Símbolos: triángulos rellenos $\Delta$ para pegmatita de La Isla, cruces + para pegmatita de Enio, círculos rellenos • para pegmatita de Achio y Fermín, cuadrado relleno $\mathbf{\square}$ para pegmatita de Helio, rombo relleno $\bullet$ para pegmatita Golconda. 
entre el orden estructural relacionado con la triclinicidad medido con difracción de rayos- $X$ por el parámetro $S_{2}(4)$ y el orden $\mathrm{Si}$ / Al local medido con los espectros de ${ }^{27} \mathrm{Al}$ RMN por la anchura de la señal. En esta figura se observa todo el rango del ordenamiento $\mathrm{Si} / \mathrm{Al}$ en las posiciones tetraédricas y sirve para localizar el grado de evolución macroestructural asociado a cada una de ellas.

La Fig. 3 presenta las macroestructuras que aparecen en los dos extremos de la Fig. 2, esto es: (i) una microclina desordenada (FB37), que está íntegramente formada por la variedad microclina alta con total desorden local en la distribución $\mathrm{Si} / \mathrm{Al}$, en la que no se aprecia ningún contraste relacionado con maclado en la escala óptica (Fig. 3a), y (ii) una microclina ordenada (GcI) mayoritariamente compuesta por la variedad microclina baja, en la que se aprecia claramente una macroestructura formada por una multiplicidad de distintos tipos de dominios-macla que siguen las leyes de la Albita separados por paredes en posición N-S y de la Periclina con paredes en posición E-W. En el primer caso, el ángulo de extinción relativo a la traza del plano (010) en la sección (001) es completamente homogéneo con un valor de alrededor de $6^{\circ}$. Cuando estas muestras de baja triclinicidad se estudian por microscopía electrónica de transmisión se observa la típica microestructura "tweed" (10). En el segundo caso, este mismo ángulo también es bastante homogéneo y alcanza valores cercanos a los $16^{\circ}$.
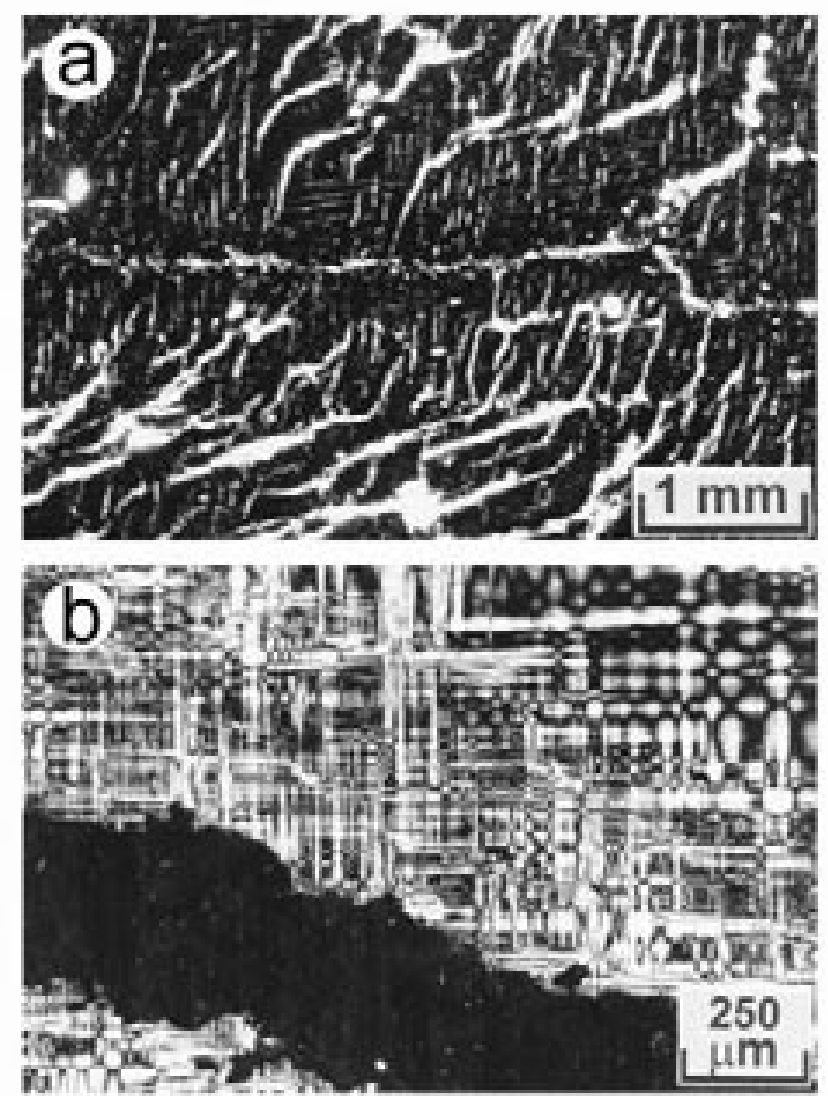

Fig. 3.-Comparación de las macroestructuras de los extremos microclina ordenada y desordenada. a) Micrografía obtenida por microscopía óptica con nícoles cruzados en la muestra FB35 de la pegmatita de La Isla. b) Idem en la muestra GcF4 de la pegmatita Golconda III.

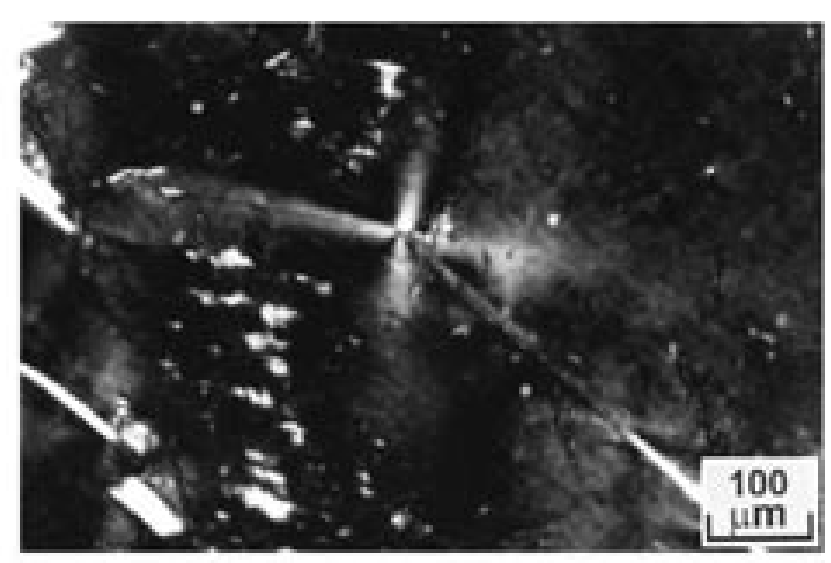

Fig. 4.-Micrografía obtenida por microscopía óptica con nícoles cruzados en la muestra En7 de la pegmatita de Enio. Obsérvese la disposición de microclina más ordenada según una estrella de cuatro puntas en el seno de una matriz de microclina alta en extinción óptica.

La Fig. 4 presenta una muestra formada mayoritariamente por microclina alta (en extinción óptica), en la que aparece una vena de feldespato sódico (orientada según NW-SE) cuya punta lateral terminal actúa como lugar de nucleación de microclinas de más alta triclinicidad. A partir de este punto de alta deformación estructural, se propaga la transformación según cuatro frentes según las direcciones [010] y [100] en los que aparece microclina de mayor orden $\mathrm{Si} / \mathrm{Al}$ (zonas claras que transmiten la luz polarizada en la Fig. 4). Las zonas recristalizadas dibujan un conjunto a modo de "estrella" formada por cuatro brazos o curvas sólidas de forma parabólicaexponencial en esta sección. En el interior de estas regiones en cercanía al origen, el ángulo de extinción es máximo (de unos $16^{\circ}$ en la microclina baja), disminuyendo progresivamente hacia las partes externas longitudinal y transversalmente de cada uno de los brazos hasta igualarse con el de la microclina de baja triclinicidad en extinción. La zona limítrofe en que se igualan las estructuras es muy difusa y constituye la posición

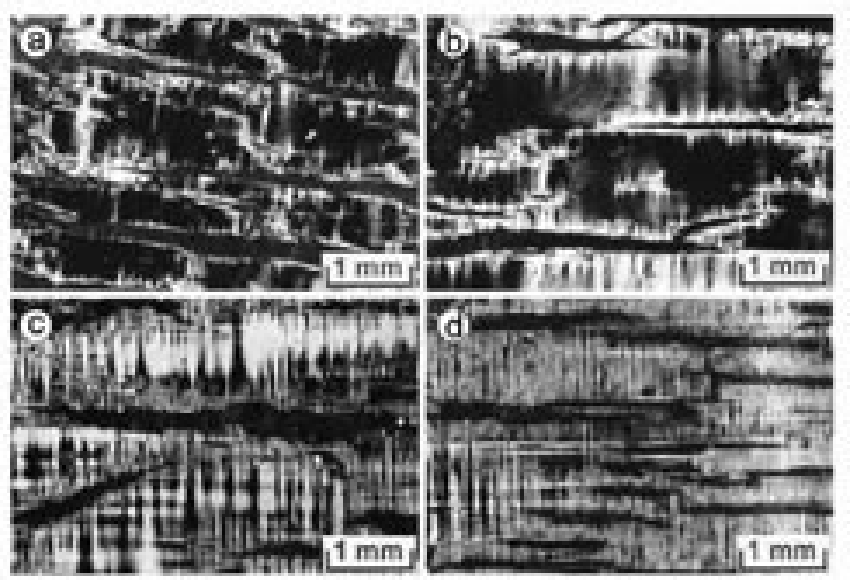

Fig. 5.-a) Micrografía obtenida por microscopía óptica con nícoles cruzados en la muestra En17 de la pegmatita de Enio. b) idem muestra F2b de la pegmatita de Fermín. c) idem muestra Ac12 de la pegmatita de Achio. d) idem muestra Dlr1 de la pegmatita Helio. 
del frente de transformación. En este trabajo, se considera una "avalancha" de recristalización al volumen de microclina más ordenada comprendido entre el punto de nucleación y el frente de transformación. Hay que destacar que no se han observado estos fenómenos de transformación a partir de las interfases semi-coherentes con las películas de feldespato sódico de las texturas micropertíticas, ni en zonas estructurales homogéneas y sin defectos, poniendo de manifiesto que la transformación tiene lugar principalmente por nucleación heterogénea.

Las micrografías de las Figs. 5 a y b pertenecen a microclinas desordenadas formadas en un régimen estático (4). En la Fig. 5a se observa como la recristalización transcurre por extensión progresiva de avalanchas de microclina ordenada a costa de la microclina desordenada. En esta figura se aprecia la superposición lateral a lo largo del recorrido, de las interfases con las venas de feldespatos sódico macropertítico, en forma de numerosas avalanchas de alcance variable. Se extienden según la dirección [100] y dan lugar a una distribución irregular pseudo-periódica de macro-estructuras "en peine" (12). La extensión longitudinal de las avalanchas no guarda relación alguna con el grosor de las venas, pero si con la distancia entre dos interfases de nucleación. Así, cuando dos venas están próximas, la extensión en que se aprecia la formación de microclina de mayor triclinicidad está generalmente más desarrollada. Cabe destacar que cuando se solapan dos avalanchas de igual orientación estructural pero de sentido de propagación contrario (es decir, generadas en dos interfases distantes y opuestas una en frente de la otra) ocurren fenómenos de coalescencia entre ellas.

Las avalanchas que aparecen en la microclina de la Fig. $5 \mathrm{~b}$ tienen mayor extensión longitudinal que las mostradas en la Fig. 5a. Además, el área con un ángulo de extinción de microclina cuya distribución $\mathrm{Si} / \mathrm{Al}$ está completamente ordenada es también mayor. El ángulo de extinción varía gradualmente desde las zonas de máximo orden hasta las zonas desordenadas que mayoritariamente forman esta imagen. En la figura se puede apreciar que se empiezan a formar conexiones entre avalanchas generadas en lugares separados por grandes distancias cruzando las regiones de baja triclinicidad en extinción óptica. Por otro lado, es evidente que las avalanchas formadas a un lado y a otro de una misma vena tienen aspecto y extensión diferente. Esta ausencia de simetría implica claramente que durante el inicio de la recristalización, frentes desarrollados en interfases opuestas se comportan de manera independiente. Por lo tanto, a partir de la Fig. 5b se induce que en principio tales correlaciones entre avalanchas distantes entre sí debieran producirse sólo como fruto del azar, ya que las macroestructuras "en peine" en interfases opuestas no tienen igual desarrollo.

La fotomicrografía de la Fig. 5c pertenece a una microclina transicional (4) cuyo volumen de microclina baja tienen mayor extensión que el de microclina alta, y por tanto la proporción se ha invertido respecto a la muestra anterior. Las regiones de microclina alta forman zonas irregulares que están aisladas y distales respecto de las interfases con las venas macropertíticas. Se aprecian dominios separados por paredes que limitan regiones en cuyo interior se da un contraste más homogéneo que en las avalanchas de las muestras anteriores. La orientación relativa entre dominios se puede correlacionar con las leyes de la Albita y de la Periclina, y por tanto se trata de dominios-macla que se han formado por individualización de las avalanchas. Sin embargo, los límites entre dominios son más netos en las zonas proximales a las interfases con las venas y se vuelven más difusos a medida que aumenta la distancia desde éstas. Obsérvese en esta mesoestructura ahora son las paredes de dominio (o sus prolongaciones difusas más o menos onduladas) relacionadas con distintas interfases de nucleación las que tienden a ser coincidentes. En esta misma figura se observa como en las zonas distales a las interfases aparecen patrones ortogonales por interferencia entre frentes de transformación por intersección a unos $90^{\circ}$ para dar patrones tipo "tablero de ajedrez" (7).

Finalmente, la Fig. 5d ha sido obtenida en una microclina ordenada formada en un régimen dinámico (4) que ha recristalizado prácticamente en su totalidad, y por tanto está formada por microclina baja de triclinicidad máxima. La macroestructura consiste en una alternancia transversal regular de dominios-macla con morfología laminar, límites paralelos y extinción homogénea, maclado "polisintético" (2), que en este caso particular están orientados principalmente según la ley de la Albita. Las paredes de dominio están en general perfectamente definidas y su tamaño longitudinal se extiende desde una interfase hasta la opuesta más próxima. Cabe destacar la regularidad en el tamaño de dominio o equidistancia entre paredes de dominio-macla, de forma que la distancia transversal promedio varía con la distancia longitudinal a las interfases con el feldespato sódico, variando el grosor de los dominios entre 10 y $100 \mu \mathrm{m}$. Cuando dos interfases están cercanas, la periodicidad es menor, y más regular al tiempo, que las paredes de dominio son más rectas y netas que cuando las interfases están más distantes entre sí, en cuyo caso se aprecian paredes más onduladas así como mayores periodicidades y peor definición. En consecuencia, la regularidad de esta macroestructura parece responder a un carácter heredado de la distribución de las avalanchas previamente formadas a lo largo del recorrido de las interfases con las venas.

En la parte inferior de la micrografía de la Fig. 6a se observa una vena de feldespato sódico con maclado polisintético según la ley de la Albita cuyas paredes de dominio coinciden parcialmente con las paredes de dominio según la misma ley en la microclina. En ocasiones los límites entre dominios se prolongan cruzando las interfases feldespato sódico-potásico. El patrón de maclado de la microclina evidencia un marcado carácter pseudo-periódico según la misma ley. La alternancia de contraste claro-oscuro se debe a la diferente orientación de los dominios que implica un ángulo de extinción ligeramente distinto. En la Fig. 6 b se presenta un mapa químico del contenido en $\mathrm{Na}$ obtenido por microsonda electrónica en la región central de los dominios-macla de la Fig. 6a. Las exsoluciones de feldespato sódico micropertítico, que se distinguen claramente por su mayor contenido en $\mathrm{Na}$ (color blanco), presentan grados de deformación variable. En esta figura se observa que hay lamelas de feldespato sódico con distinto grado de deformación plástica y otras claramente divididas por rotura. Obsérvese que los lugares en los que se distingue mayor deformación son las áreas cercanas a las paredes de los dominio-macla de la microclina, que separan las partes fracturadas de los dominios de feldespato sódico. El mapa de contenido en sodio indica que en tales límites se dan concentraciones anómalamente altas de este catión en relación a las partes internas de los dominios. Este hecho se puede relacionar con zonas estructurales que contienen una mayor densidad de defectos cristalinos capaces de incorporar este catión. Esta microtextura evidencia claramente su origen mecánico, producida en eventos que son posteriores tanto a las exsoluciones de feldespato sódico como a la configuración regular de los dominios-macla del feldespato potásico. 

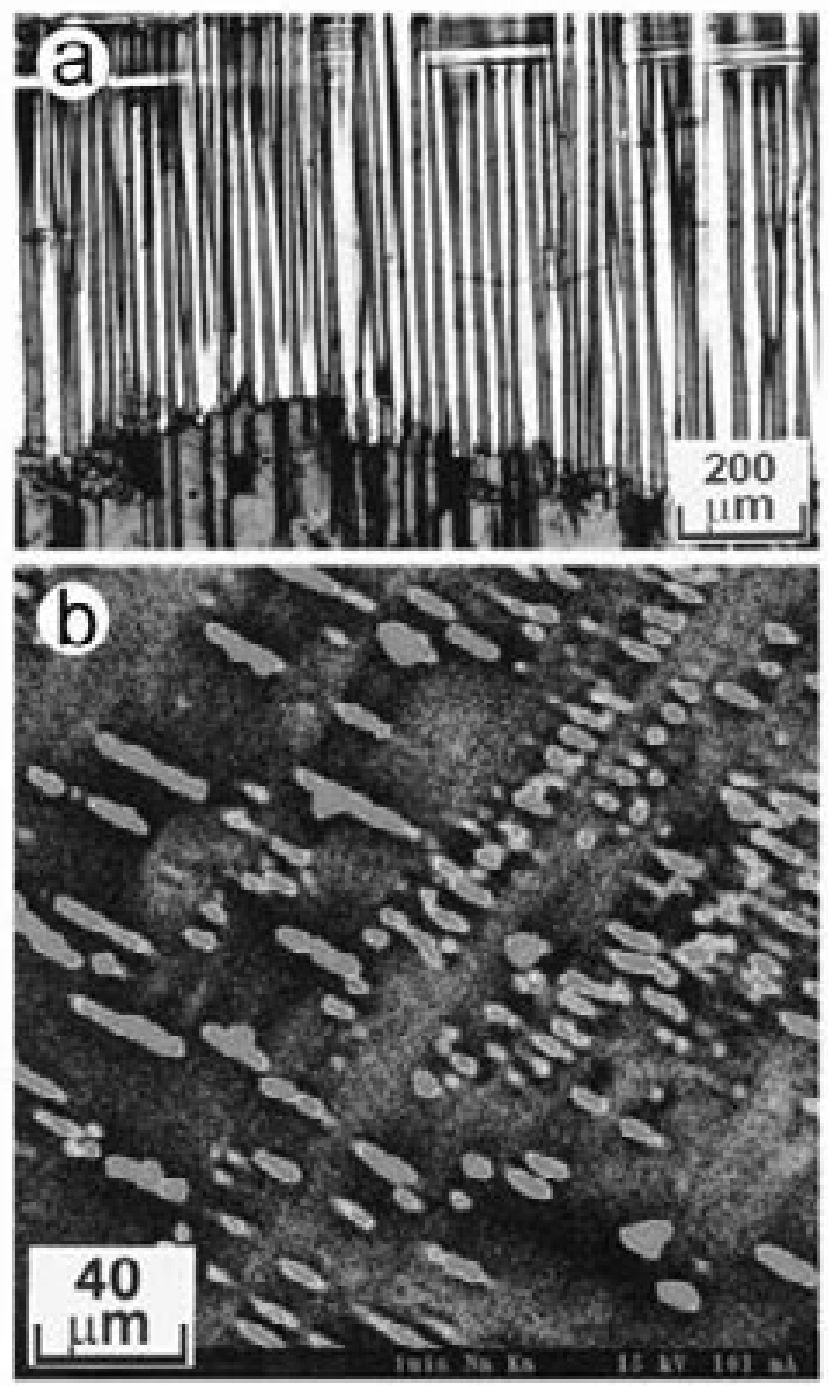

Fig. 6.- a) Micrografía obtenida por microscopía óptica con nícoles cruzados en la muestra GcInt4Ab (GcI) de la pegmatita Golconda III. El mapa químico de Na se ha realizado en la parte central de esta superficie. b) Mapa químico de la concentración en Na. Obsérvese la deformación plástica de la textura.

En la Fig. 7a los dominios finos en película de feldespato sódico micropertítico aparecen fracturados, y están tanto más fuertemente divididos cuanto más cerca se encuentran de las interfases con las venas. Se aprecia una importante reorientación de los dominios de microclina según la ley de la Albita a modo de bandas de deformación que quedan marcados por las películas de feldespato sódico en zig-zag. También se advierte otra característica común a la mayoría de las macroestructuras observadas en las microclinas bajas, que consiste en que las paredes de dominio pueden presentar un aspecto difuminado. La resolución por microscopía electrónica en otras muestras indica que se trata de un maclado submicroscópico de sustitución (7). El maclado polisintético según la ley de la Albita con orientación N-S en la microclina de la Fig. $7 \mathrm{~b}$ está parcialmente sustituido por planos de tipo Periclina con orientación E-W. Esta superposición desdibuja la regularidad macroestructural y genera otra variedad de patrones de maclado diferentes. Cuando las macroestructuras de sustitución son suficientemente gruesas

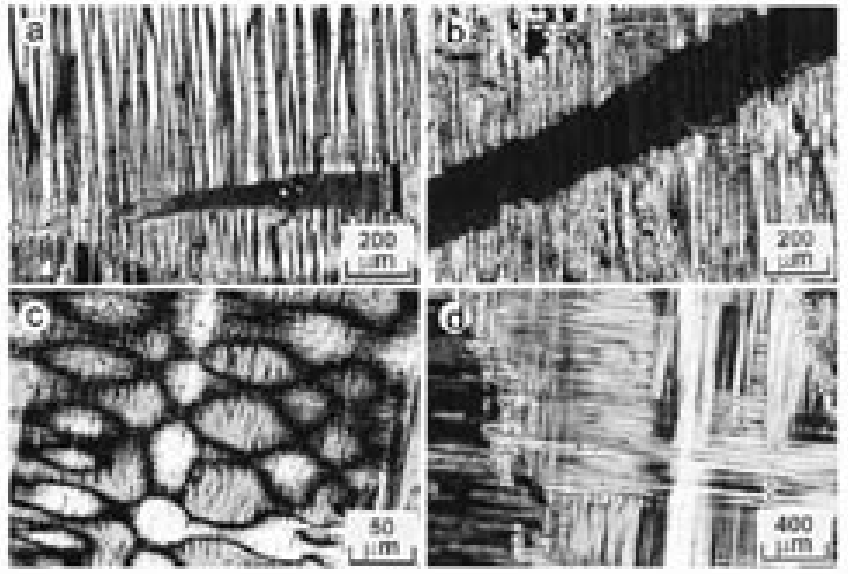

Fig. 7.- a) Micrografía obtenida por microscopía óptica con nícoles cruzados en la muestra Dlr8 de la pegmatita Helio, donde los límites entre dominios macla relacionados por la ley de la Albita son coincidentes con límites de bandas de deformación que quedan marcadas por los zig-zags de dominios en película de feldespato sódico. b) idem de la muestra GcF5 en donde se aprecian como las paredes de tipo Periclina (según E-W) remplazan las de tipo Albita (según N-S). Una vena de feldespatos sódico cruza la micrografía de SW a NE. c) idem de la muestra Dlr8 en donde se aprecia un patrón en enrejado fino que coincide con los límites entre dominios en un patrón en tablero de ajedrez. d) idem de la muestra GcInt2.

se puede obtener resolución a la escala óptica, apreciándose un maclado en "enrejado" $(2,7)$ de mayor tamaño, como el que se sobreimpone al patrón en "tablero de ajedrez" de la Fig. 7c. En la Fig. 7d se distinguen regiones con patrones formados por dominios-macla de distribución pseudo-periódica que siguen tanto la ley de la Albita en orientación E-W como de la Periclina con orientación N-S, cuya intersección genera de dominios en ángulo recto que evolucionan a dominios en aguja $(13,14)$. Esta macroestructura presenta distintos grados de descomposición, bien por desaparición aparente de los límites, bien por sustitución por un maclado en enrejado muy fino, o bien por su destrucción ligada a un engrosamiento con texturas turbidíticas. Este último caso aparece en las interfases entre las venas de feldespato sódico y la matriz potásica, debido a la circulación de fluidos acuosos de muy baja temperatura, como se aprecia a la izquierda de la Fig. 7d.

En la parte central de la Fig. 8a se observan grandes dominios tipo Periclina de extinción homogénea a unos $18^{\circ}$, es decir compuestos por microclina totalmente ordenada con máxima triclinicidad, cuyas paredes tienen localmente aspecto difuminado. En la parte superior, estos dominios están transformados en una mesoestructura "serrada", similar a otras muestras estudiadas por TEM (7). El mapa químico de Na de la parte central de la imagen (Fig. 6b) muestra que el contenido en $\mathrm{Na}$ de la microclina es mas alto (tono claro) y tiene una distribución más homogénea en las partes internas de los dominios centrales que en sus bordes o en el resto de la misma (tonalidad más oscura). Las paredes de dominio con orientación aproximadamente N-S aparecen como fracturas (en color negro) en las que localmente se desarrollan dominios muy delgados de feldespato sódico en película (líneas finas blancas) que tienen formas rectas, grosor constante y grandes longitudes en relación al espesor. Obsérvese una familia de fracturas más o menos rectas con orientación aproximada E-W que cortan las fracturas N-S. La parte superior serrada 

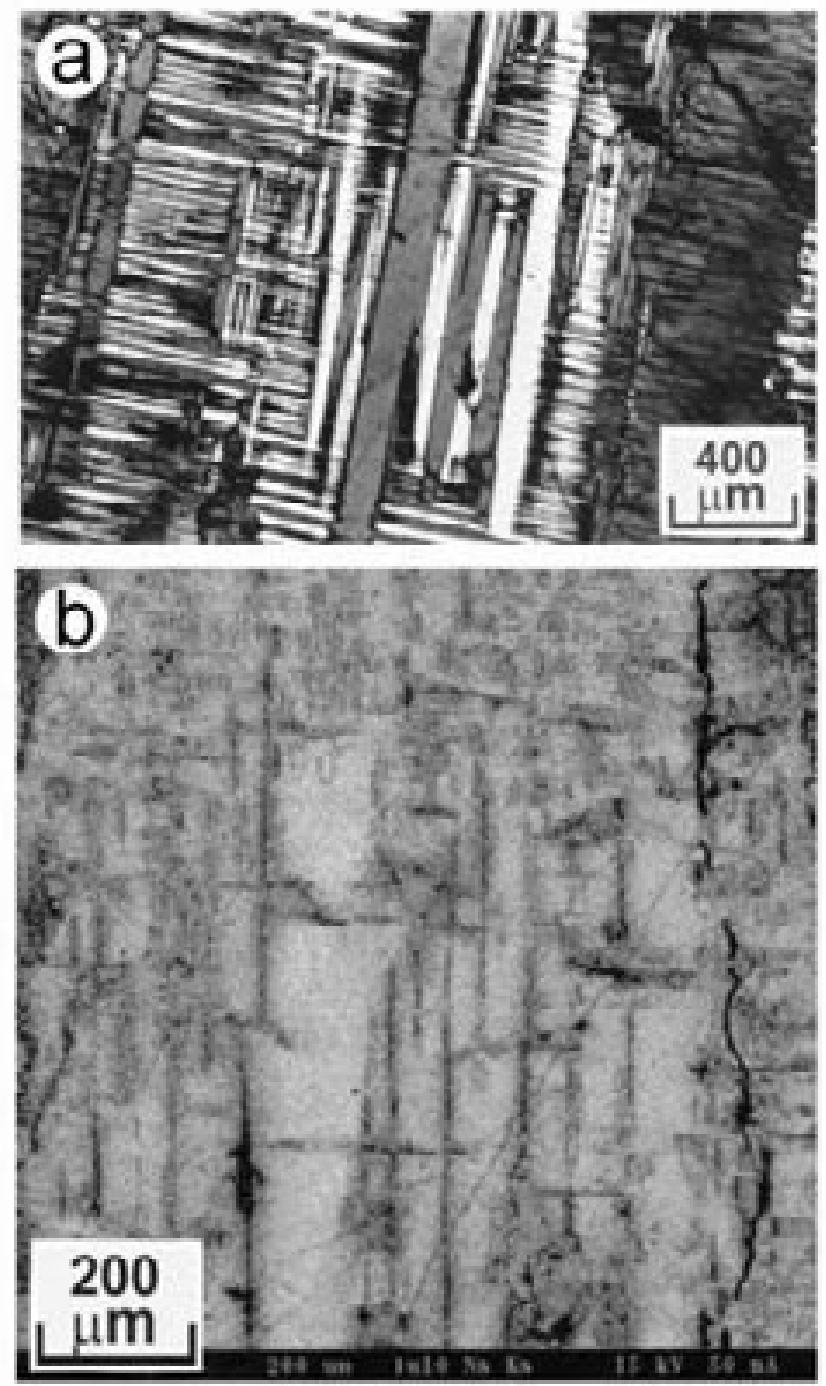

Fig. 8.- a) Micrografía obtenida por microscopía óptica con nícoles cruzados en la muestra GcInt4Ab (GcI), apreciándose dominios según la ley de la Periclina orientados según aproximadamente N-S, siendo especialmente claros en la parte central de la imagen, en la que se ha realizado un mapa químico de contenido en $\mathrm{Na}$. b) Mapa químico de Na mostrando, distintas fracturas, dominios de feldespato sódico en blanco con distintas morfologías, y la matriz de feldespato potásico en la que la concentración en Na es variable.

aparece como resultado de deformación plástica, ya que las películas de feldespato sódico están rotas y divididas por planos coincidentes con los límites entre dominios de tipo Albita, evidenciando la importancia de los esfuerzos mecánicos. La fractura irregular gruesa de la parte derecha está parcialmente rellena de feldespato sódico (manchas en blanco) y su recorrido coincide con zonas en las que se difumina el maclado polisintético regular de la Fig. 8a. Esta imagen indica que la circulación de fluidos a través de (i) fracturas desarrolladas a partir de límites previos entre dominios o (ii) fracturas irregulares; produce la extracción de $\mathrm{Na}$ de la estructura de la microclina, que está asociada con la destrucción de las interfases de las macroestructuras regulares.

\section{DISCUSIÓN}

\subsection{Línea común de evolución en microclinas}

La existencia de una línea común de evolución en los feldespatos pertíticos ya ha sido reconocida en pegmatitas graníticas $(10,15)$ y en otras rocas (16), y se manifiesta tanto en las características estructurales (por aumento de la triclinicidad en la fase del feldespato potásico), como en las texturales (principalmente por la disposición geométrica y el tipo de interfase entre la fase matriz y la fase huésped, y por el engrosamiento de esta última). Su causa termodinámica última es el re-equilibrado relacionado con el descenso en energía libre del cristal, ligado al ordenamiento $\mathrm{Si} / \mathrm{Al}$, a la disminución de energía acumulada en los límites entre dominios de igual estructura pero distinta orientación (planos de macla), así como con límites de diferente composición química o distinta fase (interfases) (16). El proceso de evolución opera mediante recristalización y su intensidad depende de los factores cinéticos del sistema durante el enfriamiento para generar pseudomorfos (17).

Este trabajo ha permitido distinguir dos etapas sucesivas e independientes de recristalización: la primera produce el ordenamiento local en la distribución Si / Al y la creación de patrones regulares en la macroescala, mientras que la segunda destruye la regularidad macroestructural anteriormente construida.

La etapa de construcción consiste en la ordenamiento Si / $\mathrm{Al}$ a modo de avalanchas de transformación que evolucionan progresivamente hasta estructuras regulares en dominiosmacla, siendo esta la unidad básica de construcción de la macroestructura. Se ha apreciado que la etapa tiene un desarrollo marcadamente diferente en feldespatos que evolucionan en distinto ambiente geológico. En cada régimen de evolución se generan microclinas características (4) y macroestructuras asociadas, como se expone a continuación.

En el régimen estático, las estructuras tienen evolución muy limitada apreciándose la formación y coalescencia de avalanchas de recristalización de microclina con orden $\mathrm{Si} / \mathrm{Al}$ de alta triclinicidad en el seno de una estructura homogénea en la macroescala con desorden Si/Al y baja triclinicidad a nivel local. Las microclinas más ordenadas se forman en las interfases incoherentes con las venas macropertíticas de feldespato sódico por nucleación heterogénea y las transformaciones de disparan a modo de avalanchas a partir de éstas, formando macroestructuras "en estrella" y "en peine". En este régimen, cuando dos interfases incoherentes están muy cercanas se forma cierta extensión de microclina con alto grado de ordenamiento Si/Al. Sin embargo, esto no implica necesariamente que se desarrollen los patrones en dominios-macla regulares en la macroescala, como aquellos de la microclina baja en el régimen dinámico, sino que la estructura a gran escala permanece desordenada.

Los estadios estructurales que precenden al régimen dinámico se pueden observar en el régimen transicional. En éste, se generaliza la coalescencia de avalanchas con igual variante orientacional para después individualizarse como dominios-macla incipientes y también aparecen patrones de interferencia a $90^{\circ}$ en macroestructuras en "tablero de ajedrez".

El régimen dinámico se caracteriza por la formación de patrones macroestructurales regulares en microclinas con orden local completo en la distribución $\mathrm{Si} / \mathrm{Al}$, en forma de maclas polisintéticas (que siguen tanto la ley de la Albita 
como de la Periclina y en un rango variable en la perfección de la periodicidad) que normalmente no intersectan sino que se yuxtaponen, y reorganizan para formar maclas en ángulo recto y en aguja.

La segunda etapa tiene una capacidad más limitada para generar orden $\mathrm{Si} / \mathrm{Al}$ local en las posiciones tetraédricas, pero intensa en la destrucción de la regularidad macroestructural anteriormente construida. En esta se genera la deformación plástica de los dominios con aparición paredes de dominio "serrados", bandas de deformación, maclado de relajación de límites de dominio por maclado "en enrejado" de substitución. Este último proceso podría estar ligado a la extracción de $\mathrm{Na}$ de la microclina para formar parte de las exsoluciones de feldespato sódico, y aparenta surgir desde la escala submicroscópica por engrosamiento. Además, a más baja temperatura tiene lugar la destrucción de la estructura a gran escala por interacción con fluidos acuosos tardíos que circulan a través de una red formada por la porosidad interconectada, fracturas y límites entre dominios convertidos en superficies de circulación. Durante la segunda etapa también podría producirse la reorientación de los dominios de tipo Periclina hacia orientación de tipo Albita, teniendo en cuenta la reconocida escasez de los primeros en la microclina baja $(2,7)$.

Los datos presentados indican que las tensiones mecánicas de cizalla estimulan el ordenamiento local por recristalización según campos de deformación dúctil (avalanchas) con la ayuda catalizadora de unas pocas moléculas de agua que se dan en el medio por difusión volumétrica, ya que los fluidos acuosos residuales circulan principalmente por las interfases. Esta conjunción debe hacer posible la construcción progresiva de la regularidad hasta que se completan los patrones en dominios-macla en la macroestructura, mientras exista cierto desorden en la distribución Si/Al. Una vez que se completa el ordenamiento, los esfuerzos mecánicos posteriores primero producen deformaciones localizadas en los límites entre dominios y más tarde, destrucción de la regularidad por deformaciones plásticas, como sugieren las fotomicrografías y los mapas químicos de sodio (Fig. 6 a 8).

\subsection{Recristalización auto-organizada con patrones macroestructurales}

Si no actúan los factores cinéticos, el ordenamiento de los cationes tetraédricos en la distribución $\mathrm{Si} / \mathrm{Al}$ de la microclina desordenada no parece ocurrir de manera espontánea ni para velocidades de enfriamiento de poco grados centígrados por millón de años $(16,17)$. Las microclinas desordenadas pueden ser mayoritarias incluso cuando estos factores están presentes como en las rocas pegmatíticas, indicando que tales factores deben actuar de manera sinérgica para producir microclina ordenada. Una explicación habitual es que el descenso en energía libre del cristal por orden $\mathrm{Si} / \mathrm{Al}$ es muy inferior a la energía de activación necesaria relacionada con la rotura de enlaces Si-O y Al-O. Además, la formación de dominios con secuenciación orden-antiorden podría ser un carácter adicional que inhibe el engrosamiento de los dominios (18). Por otro lado, la energía liberada por el ordenamiento parece ser igual a la necesaria para forzar a los cristales a retener su morfología original durante el proceso (19).

La formación de microclinas perfectamente ordenadas a nivel local que manifiestan además macroestructuras perfectamente periódicas y regulares según el proceso de evolución estructural por recristalización que se expone en este trabajo es inexplicable si se analiza desde el aquel punto de vista, ya que requiere la coincidencia en la rotura y el ensamblaje de un número enorme de enlaces de alta energía y largos tiempos de vida media. Desde este punto de vista, la aparición de estos patrones regulares a gran escala resulta ser un carácter emergente completamente imprevisible e improbable cuando se analiza la transformación de las microclinas tan sólo desde el punto de vista de la rotura local de enlaces. Para explicarlo se hace necesario apuntar la existencia de un comportamiento cooperativo global, en el que participe un importante volumen edificio cristalino en base a procesos de auto-organización (20). En este contexto, el efecto catalítico de fluidos acuosos como agente catalizador y el efecto acumulativo por estimulación energética externa procedente de múltiples ciclos de esfuerzos tectónicos de cizalla del régimen dinámico resultan como condiciones necesarias.

\subsection{Pertitas como sistemas complejos}

La descripción del proceso evolutivo de microclinas resulta más completo cuando se correlaciona la recristalización a nivel local por ordenamiento $\mathrm{Si} / \mathrm{Al}$ con la formación de patrones macroestructurales, en comparación a cuando se realiza una descripción más simple en términos termodinámicos de transformación de fase. Este estudio pone de manifiesto las limitaciones que tiene el concepto de "fase de Gibbs" en termodinámica en los minerales aquí estudiados, como ya puso Laves de manifiesto en otros feldespatos de tipo Piedra Luna (21). Además tras la existencia de microclinas transicionales subyace la dificultad práctica en la distinción entre "fases posibles" y "fases estables" $(5,18)$. Estas observaciones plantean otros interrogantes: ¿cuanto tiempo tiene que pasar para dejar de considerar "metaestable" a una estructura y considerarla perfectamente estable? ¿qué papel termodinámico juegan los defectos como características de no-equilibrio? ¿cuáles son las características esenciales de la identidad de un cristal?.

La incapacidad de las estructuras para re-equilibrarse, excepto en condiciones excepcionales, el comportamiento de sistema abierto al flujo de moléculas de agua y el efecto estimulo de la presión tectónica, la formación de avalanchas de transformación estructural y la emergencia de patrones macroestructurales por auto-organización describen las pertitas como sistemas complejos $(22,23)$. En consecuencia, las microclinas son cristales que pueden manifestar un comportamiento cooperativo y coherente a escala global en su estructura, y donde los defectos no son simples errores estructurales sino elementos esenciales de la complejidad del cristal.

\section{CONCLUSIÓN}

El estudio de microclinas pertíticas de pegmatitas graníticas mediante microscopía óptica, difracción de rayos- $X$, resonancia magnética nuclear de alta resolución del núcleo ${ }^{27} \mathrm{Al}$, y de la distribución de Na por mapas químicos por microsonda electrónica han permitido describir estas pertitas como sistemas complejos. Durante el enfriamiento se distinguen dos periodos contrastados, uno de contrucción y otro de destrucción, en la evolución estructural de microclinas pertíticas. El periodo de construcción de los patrones macroestructurales de maclado es una consecuencia del progresivo ordenamiento no-ergódico en las posiciones tetraédricas de los átomos de $\mathrm{Si}$ y $\mathrm{Al}$ a 
nivel local. Estos patrones surgen del auto-ensamblado de los frentes de transformación (avalanchas) que se disparan desde las interfases incoherentes con las venas de feldespato sódico. Según esta descripción las pertitas sufren procesos de auto-organización estructural gracias al estímulo de la energía tectónica de cizalla y al efecto catalítico de moléculas de agua distribuidas a través de todo el volumen del cristal. El periodo de destrucción está relacionado con otros patrones característicos que restan regularidad.

\section{AGRADECIMIENTOS}

Agradecemos al geólogo brasileño Odulio Marensi de Moura su gran ayuda con las pegmatitas de Brasil, a Martín Fernández Hernán con las pegmatitas españolas y al proyecto C.I.C.Y.T. CGL2004-03564/BTE y a la Beca Postdoctoral del MEC de estancia en el CNRS (Orleáns, Francia).

\section{BIBLIOGRAFÍA}

1. L. Sánchez-Muñoz; J. García-Guinea; J. Sanz; J.B. Carda Castelló. Feldespatos, materias primas fundentes y otros materiales afines. En: Cap. X de Enciclopedia Cerámica Tomo 2.2: Materias Primas y Aditivos Cerámicos, L. Sánchez-Muñoz y J.B. Carda Castelló, Faenza Editrice Ibérica, S.L., Castellón, 2003.

2. J.V. Smith, W.L. Brown. Feldspar Minerals, $2^{\text {nd }}$ edition, Springer-Verlag, Berlin, 1988.

3. R.A. Yund RA. Microstructure, kinetics and mechanisms of alkali feldspars exsolution. Rev. Mineral. 2, 177-202, 1983.

4. L. Sánchez-Muñoz, J. García-Guinea, V. Correcher, J. Sanz. Regímenes estático y dinámico en la evolución estructural de microclinas pertíticas. Bol Soc Esp Ceram V., 45, [5] 289-299, (2006)

5. H.U. Bambauer, C. Krause, H. Kroll. TEM-investigation of the Sanidine/ Microcline Transition Across Metamorphic Zones: the K-feldspar Varieties. Eur. J. Mineral. 1, 47-58, (1989).

6. L. Sánchez-Muñoz, L. Nistor, G. Van Tendeloo, J. Sanz. Modulated Structures in $\mathrm{KAlSi}_{3} \mathrm{O}_{\mathrm{s}}$ : A Study by High Resolution Electron Microscopy and Si-29 MAS-NMR Spectroscopy. Jour. Electron Micros., 47, 1, 17-28, (1998).
7. J.D. Fitz Gerald, A.C. McLaren. The Microstructures of Microcline from Some Granitic Rocks and Pegmatites. Contrib. Mineral. Petrol., 80, 219-229, (1982).

8. F. Laves, J.R. Goldsmith. Polymorphism, Order, Disorder, Diffusion and Confusion in the Feldespars. Cursillos y Conferencias del Instituto Lucas Mallada, Fasc VIII, 71-80, (1961).

9. Laves F. The lattice and twinning of microcline and other potash feldspar. J. Geol. 58: 548-571, (1950).

10. L. Sánchez-Muñoz. Influencia de la exsolución Na/K sobre las características estructurales y microtexturales de feldespatos alcalinos pegmatíticos. Tesis Doctoral Universidad Complutense de Madrid, 1992.

11. D.B. Stewart, P.H. Ribbe. Optical Properties of Feldspars. Rev. Mineral 2, 121-139 (1983)

12. A.S. Marfunin. The Relation Between Structure and Optical Orientation in Potash-soda Feldspars. Cursillos y Conferencias del Instituto Lucas Mallada, Fasc VIII, 97-109, (1961)

13. E.K.H. Salje, A. Buckley, G. Van Tendeloo, Y. Ishibashi, G.L. Nord. Needle Twins and Right-Angled Twins in Minerals: Comparison Between Experiments and Theory. Am. Mineral. 83, 811-822, (1998).

14. D.C. Palmer, A. Putnis, E.K.H. Salje. Twinning in tetragonal leucite. Phys. Chem. Minerals 16, 289-303, (1988).

15. P. Cerný. Evolution of Feldspars in Granitic Pegmatites, pp 501-540 en NATO ASI Series C: Mathematical and Physical Sciences vol 421: Feldspars and Their Reactions, Ed. I. Parsons, Kluwer Academic Publishers, Dordrecht, 1993.

16. W.L. Brown, I. Parsons. Feldspars in Igneous Rocks, pp 449-499 en NATO ASI Series C: Mathematical and Physical Sciences vol 421: Feldspars and Their Reactions, Ed. I. Parsons, Kluwer Academic Publishers, Dordrecht, 1993

17. R. F. Martin. Controls of Ordering and Subsolidus Phase Relations in Alkali Feldspars. pp 313-336 en The Feldspars, Eds. W. S. MacKenzie and J. Zussman, Manchester University Press, 1974.

18. W. L. Brown, I. Parsons. Alkali Feldspars: Ordering Rates, Phase Transformations and Behaviour Diagrams for Igneous Rocks. Mineral. Magazine, 53, 25-42, (1989).

19. R.A. Eggleton, P.R. Buseck. The Orthoclase-Microcline Inversion: A HighResolution Transmission Electron Microscope Study and Strain Analysis. Contrib. Mineral. Petrol. 74, 123-133, (1980).

20. T. Dewers, P. Ortoleva. Self-organization of mineralization patterns in metamorphic rocks through mechanochemical coupling. J. Phys. Chem. 93, 2842-2848, (1989)

21. F. Laves. The Growing Field of Mineral Structures, pp 174-189, en Fifty Years of X-Ray Diffraction. IUCr XVIII Congress, Glasgow, Scotland, Ed. P.P. Ewald, 1962.

22. C.M. Cross, P.C. Hohenberger. Pattern-formation outside of equilibrium. Rev. Modern Phys., 65, 3, 851-1112, (1993)

23. A.R. Bishop, K.O. Rasmussen, J Röder, T. Lookman, A. Saxena, A. Vanossi, P. Kevrekidis. Complexity at Mesoscale. arxiv.org/PS_cache/nlin/ pdf/0012/0012050.pdf; (2000). 